\title{
Piezoelectric Relaxations in Collagen Gelatin Systems
}

\author{
Hiroyoshi UEDA and Eiichi FukadA \\ The Institute of Physical and Chemical Research, \\ Wako-shi, Saitama 351, Japan.
}

(Received January 26, 1978)

\begin{abstract}
Films consisting of three joined layers of gelatin, oriented collagen, and gelatin were prepared. The temperature dependence of piezoelectric constants $\left(d=d^{\prime}-i d^{\prime \prime}\right.$ and $e=e^{\prime}-i e^{\prime \prime}$ ) was determined at $10 \mathrm{~Hz}$ for the combined films with different hydrations. At a moisture content above $30 \mathrm{wt} \%, d^{\prime}$ and $e^{\prime}$ decreased with increasing temperature just above $-100^{\circ} \mathrm{C}$, and their signs reversed around $-30^{\circ} \mathrm{C}$, and approached zero around room temperature. The temperature dependence of the piezoelectric current induced by static stress was also determined for a film with a moisture content of about $33 \mathrm{wt} \%$. The sign reversal of the decaying current was observed at intermediate temperatures in accord with the sign reversal of $d^{\prime}$ in a.c. measurements. The theoretical explanation for this was given on the basis of the Maxwell-Wagner type dielectric relaxation in a two phase system and the decay of piezoelectric polarization in collagen layer. The same explanation may be applied for the temperature dependence of the piezoelectric constant of bone, which consists of collagen fibers and hydroxyapatite.
\end{abstract}

KEY WORDS Piezoelectric Relaxation / Collagen / Gelatin / Composite Film / Hydration / Maxwell-Wagner Relaxation /

The frequency and temperature dependence of the piezoelectric, elastic, and dielectric constants is influenced by the inhomogeneous structure in polymers. A mechanical relaxation due to the inhomogeneity in dispersed systems is called the Fröhlich-Sack effect. ${ }^{1}$ A dielectric relaxation due to the inhomogeneity is called the MaxwellWagner effect. ${ }^{2}$ The theoretical interpretation for the piezoelectric relaxations in polymers is often based upon a two phase model, consisting of the piezoelectric phase and the nonpiezoelectric phase. $^{3,4}$ A three-phase model consisting of the piezoelectric phase, the nonpiezoelectric phase and the boundary phase has been also proposed. ${ }^{5}$

Hydration in polymers influences their elastic and dielectric properties. In general d.c. conductivity in polymers increases with an increase in hydration. Some polymers change their structure due to hydration. ${ }^{6,7}$ For these reasons, the piezoelectric properties of polymer are also influenced by hydration. ${ }^{8,9}$ In order to investigate in detail the influence of hydration on the piezoelectricity of polymers, a model experiment was undertaken using the combined films of collagen and gelation of different hydrations. The combined film is a model system for the piezoelectric polymer; the collagen layer being the piezoelectric phase and the gelation layer the nonpiezoelectric phase.

\section{EXPERIMENTAL}

Combined films of collagen and gelatin were prepared in the following way. Collagen tapes, 2 -mm wide and 0.09 - $\mathrm{mm}$ thick, having been oriented longitudinally during manufacture, were joined together breadthwise to increase their width using water as an adhesive. Such a collagen film was placed on gelation gel prepared in a nylon cell, and the gelatin solution was poured over the collagen film. After drying in air for several days, a combined film consisting of parallel layers of gelatin, collagen and gelatin was obtained. The thickness of the collagen layer was approximately $0.2 \mathrm{~mm}$ and that of the gelatin layers approximately $0.05 \mathrm{~mm}$ each. A rectangular sample was then cut at 45 degrees to the orientation direction of collagen fibrils. In this sample a tensile stress along the length of the film gives a shear stress 


\section{H. UEDA and E. FuKADA}

with respect to the oriented collagen fibrils. Finally, silver was evaporated on to the central area, $5 \times 5 \mathrm{~mm}$, of both surfaces to serve as electrodes. The dimensions of the sample film was about $10 \times 5 \times 0.3 \mathrm{~mm}$.

Measurements of the piezoelectric strain constant, $d=d^{\prime}-i d^{\prime \prime}$, the piezoelectric stress constant, $e=e^{\prime}-i e^{\prime \prime}$, and the elastic constant, $C=C^{\prime}+i C^{\prime \prime}$, were carried out at $10 \mathrm{~Hz}$ in a temperature range from -150 to $+150^{\circ} \mathrm{C}$. The apparatus recorded the temperature variation of real and imaginary components of these constants. Sinusoidal stress, strain and polarization of the sample were detected by a piezoelectric ceramic element, a nonbonded type strain guage and a charge amplifier, respectively and the ratios of any two of these were determined by an operational circuit. The details have been given in a previous publication. ${ }^{10}$

The measured $d$-constant gives the ratio of the electric polarization to the mechanical stress applied at 45 degrees to the direction of fibrillar orientation. If rectangular coordinates are assigned to the sample, so that the $x$-axis is normal to the film plane and the $z$-axis is parallel to the orientation direction of collagen fibrils, the piezoelectric tensor component $d_{14}$ is twice the observed $d$-constant, where the subscript 14 indicates that the shear stress in the $y z$ plane produces the polarization in the $x$ direction. Although the sign of $d_{14}$ has been found to be negative, for convenience sake the observed $d$-constant will be shown as a positive quantity in the following descriptions.

The dielectric constant, $\varepsilon=\varepsilon^{\prime}-i \varepsilon^{\prime \prime}$, was measured at 10,20 , and $64 \mathrm{~Hz}$ in a temperature range from -150 to $+150^{\circ} \mathrm{C}^{9}$

Measurements of the static piezoelectricity were also carried out. A static stress of about $1.5 \mathrm{~kg} / \mathrm{cm}^{2}$ was applied to the sample by passing a constant d.c. current through a moving coil of the driver. The piezoelectric current induced between effectively short circuited electrodes was detected by a micromicro-ammeter (Keithley $610 \mathrm{~B}$ ) and its temporal variation was recorded with an osilloscope.

The samples were placed in desiccators at relative humidities (RH) of $93,84,75,63,43$, and $23 \%$ at $25^{\circ} \mathrm{C}$ for at least two days before measurement. The moisture contents of the sample at respective relative humidities were about $45,33,28,25,20$, and $15 \mathrm{wt} \%$.

\section{RESULTS}

Comparison of the temperature dependence of $d$-constant and $e$-constant for a collagen film (C), a combined film of collagen and gelatin (CG), and a thin piece of fish bone (FB), all equilibrated at 93-\% RH is shown in Figure 1. For all samples,
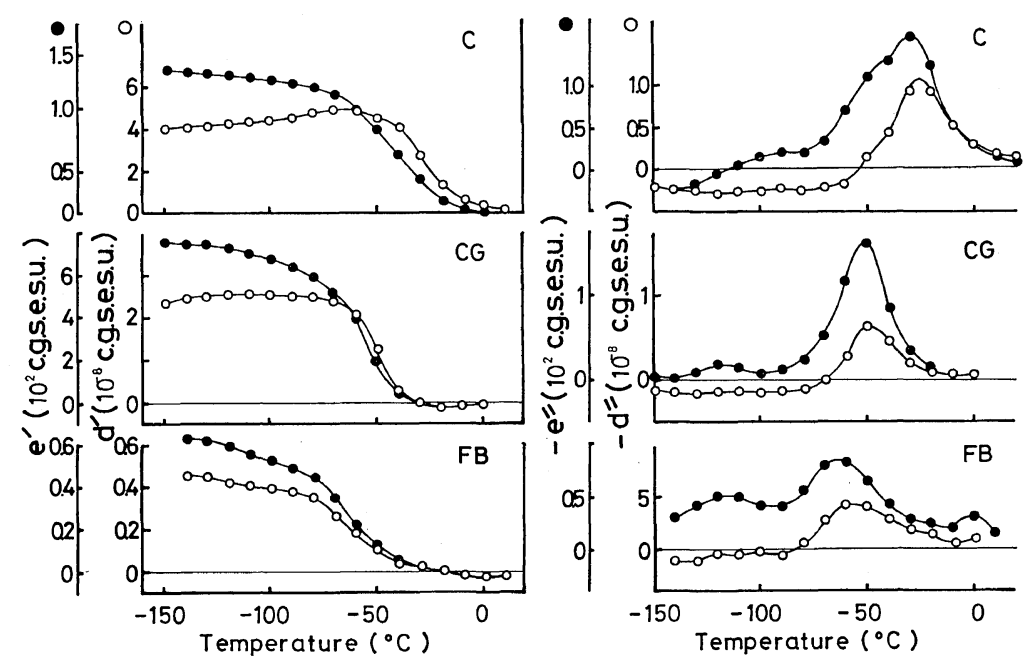

Figure 1. Temperature-dependence of the piezoelectric strain- and stress-constant, $e=e^{\prime}-i e^{\prime \prime}$ and $d=d^{\prime}-i d^{\prime \prime}$, for oriented collagen film (C), combined film of collagen and gelatin (CG), and fish bone (FB) equilibrated at relative humidity $93 \%$. 
$e^{\prime}$ decreases with increasing temperature. For collagen $(\mathrm{C}), d^{\prime}$ shows a maximum around $-50^{\circ} \mathrm{C}$ and decreases with increasing temperature. For $\mathrm{CG}$ and $\mathrm{FB}, d^{\prime}$ shows no maximum. It is noted that the sign of $d^{\prime}$ and $e^{\prime}$ for CG and FB changes from positive $\left(d_{14}, e_{14}<0\right)$ to negative $\left(d_{14}, e_{14}>0\right)$ at about $-30^{\circ} \mathrm{C}$, whereas the reversal of sign of $d^{\prime}$ and $e^{\prime}$ is not observed for collagen $\left(d_{14}, e_{14}<0\right)$. Corresponding to the change of $d^{\prime}$ and $e^{\prime}, d^{\prime \prime}$ and $e^{\prime \prime}$ of collagen (C) show a negative maximum (leading phase) around $-30^{\circ} \mathrm{C}$ and small shoulder around $-50^{\circ} \mathrm{C}$. For $\mathrm{CG}$ and $\mathrm{FB}, d^{\prime \prime}$ and $e^{\prime \prime}$ show a negative maximum around $-50^{\circ} \mathrm{C}$.

The temperature dependence of $d$-constant and $e$-constant for the combined film (CG) equilibrated at different relative humidities is shown in Figures 2 and 3. The sign reversals of $d^{\prime}$ and $e^{\prime}$ are observed only for samples equilibrated at relative humidities higher than $84 \%$.

Since the gelatin layer is considered to be a capacitor connected in series with the collagen layer, we purposely connected a capacitor of 143
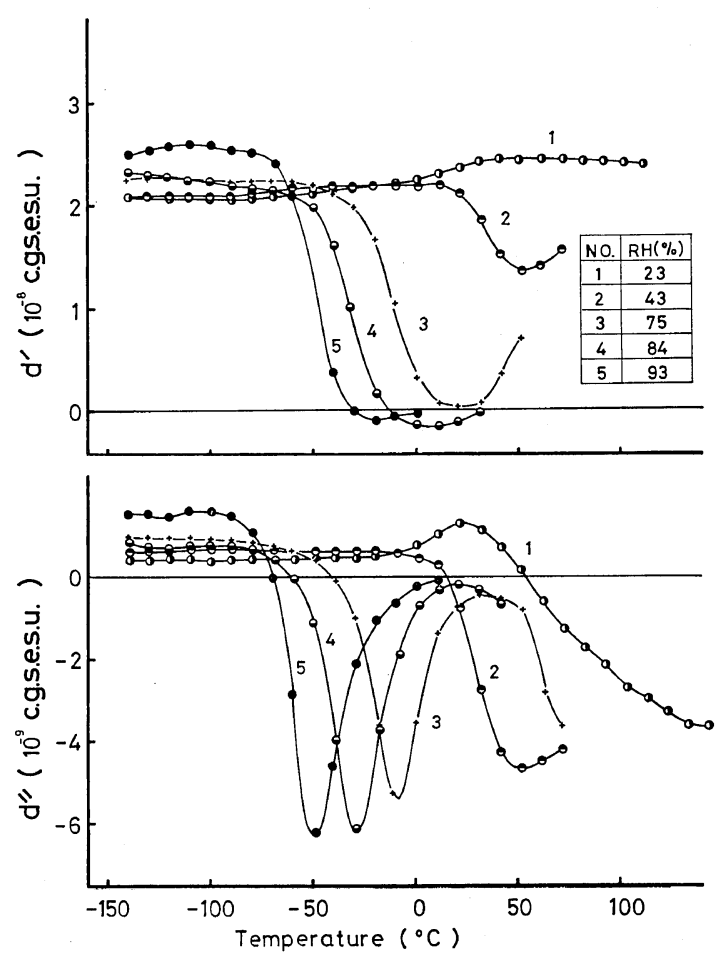

Figure 2. Temperature-dependence of the piezoelectric strain constant, $d=d^{\prime}-i d^{\prime \prime}$, for combined films of collagen and gelatin with different hydration.
pF (abbreviated to Cap in Figure 4), in series with the combined film (CG). A comparison of the
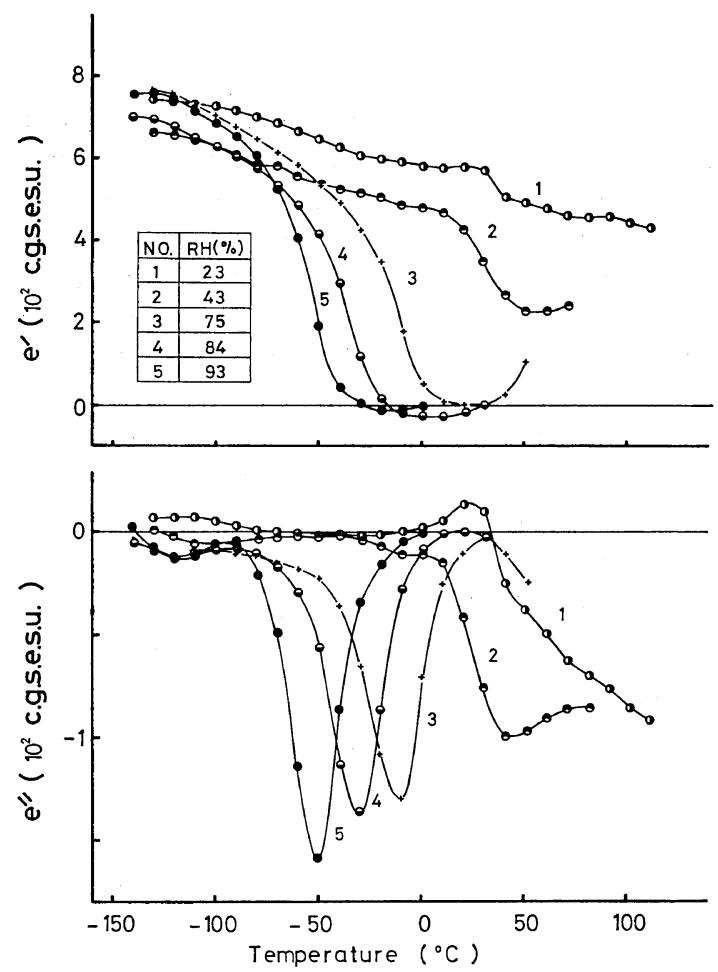

Figure 3. Temperature-dependence of the piezoelectric stress constant, $e=e^{\prime}-i e^{\prime \prime}$, for combined films of collagen and gelatin with different hydration.

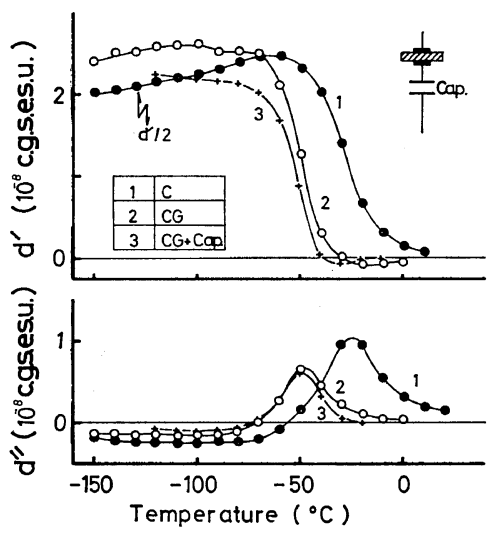

Figure 4. Temperature-dependence of the piezoelectric strain constant for oriented collagen film (C) combined film of collagen and gelatin (CG), and CG with a capacitor ( $143 \mathrm{pF})$ connected in series all equilibrated at $93-\%$ RH. 
temperature dependence of $d$-constant for $\mathrm{C}, \mathrm{CG}$, and $\mathrm{CG}+\mathrm{Cap}$, hydrated at $93-\% \mathrm{RH}$ is shown in Figure 4. It is seen that the temperature of the sign reversal of $d^{\prime}$ for $\mathrm{CG}+\mathrm{Cap}$ is lower than that for CG.

The temperature dependence of the dielectric constant at $10 \mathrm{~Hz}$ for a combined film is shown in Figure 5. For samples equilibrated at high relative humidities, $\varepsilon^{\prime}$ and $\varepsilon^{\prime \prime}$ increase sharply with increasing temperature, and become very large above $-50^{\circ} \mathrm{C}$. Figure 6 shows the frequency dependence of the dielectric constant at various temperatures between -50 and $0^{\circ} \mathrm{C}$ for a combined film equilibrated at $93-\% \mathrm{RH}$. Curves were shifted horizontally to make a single composite curve at $-20^{\circ} \mathrm{C}$ as shown in Figure 7.

Figure 8 shows the temporal variation of the piezoelectric current caused by applying a static

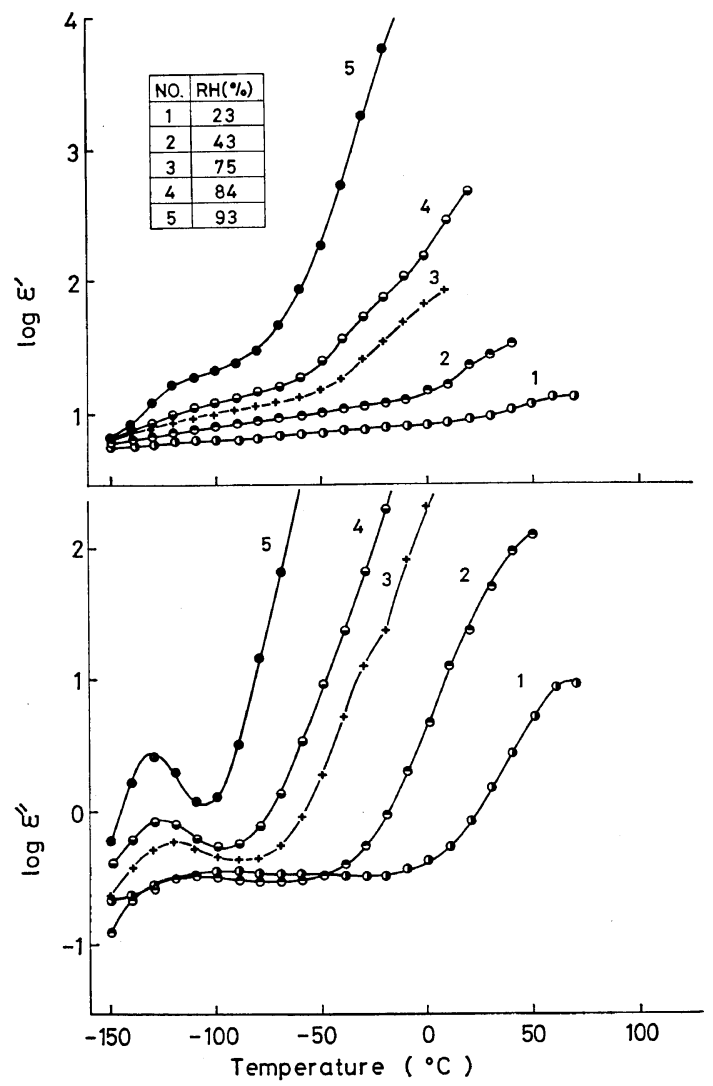

Figure 5. Temperature-dependence of the dielectric constant, $\varepsilon=\varepsilon^{\prime}-i \varepsilon^{\prime \prime}$, for combined films of collagen and gelatin with different hydration.

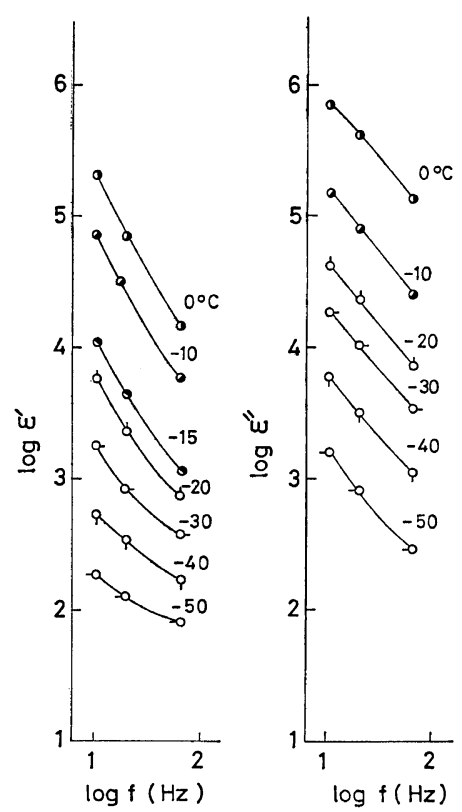

Figure 6. Frequency-dependence at different temperatures of the dielectric constant, $\varepsilon=\varepsilon^{\prime}-i \varepsilon^{\prime \prime}$, for a combined film of collagen and gelatin at 93-\% RH.

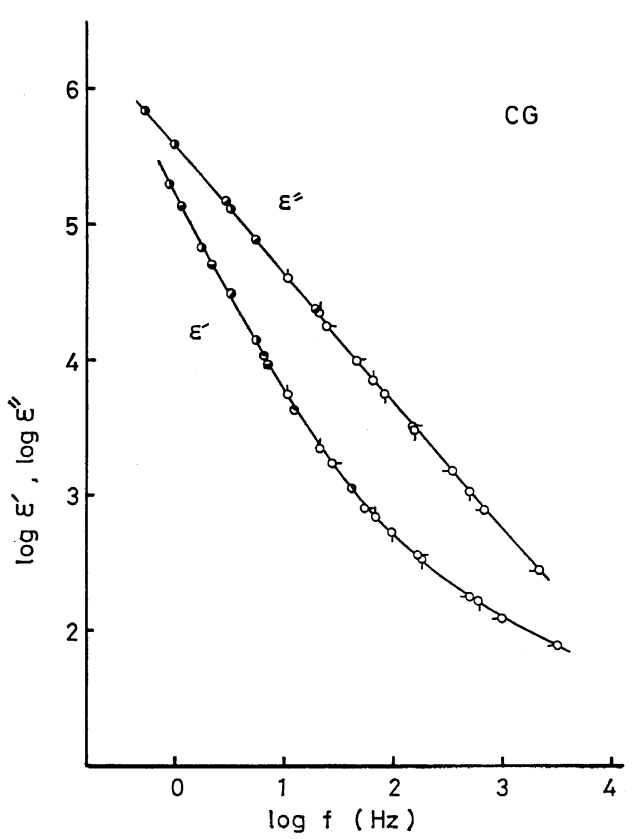

Figure 7. Master curve of the dielectric constant, $\varepsilon=\varepsilon^{\prime}-i \varepsilon^{\prime \prime}$, at $-20^{\circ} \mathrm{C}$ and $93-\% \mathrm{RH}$. 


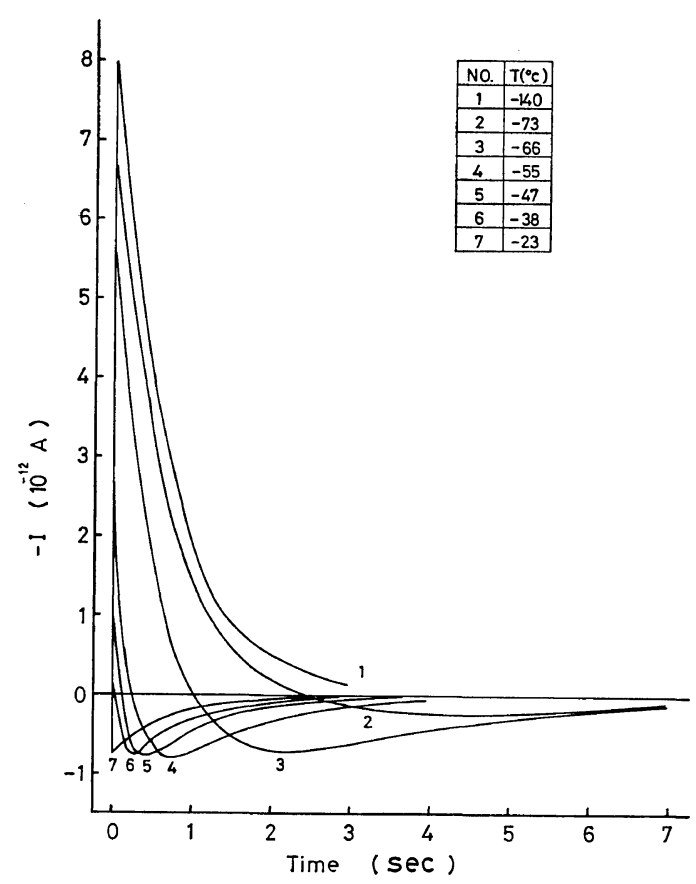

Figure 8. Variation with time at different temperatures of the piezoelectric current under a static stress for a combined film of collagen and gelatin at $84-\%$ RH.

stress of about $1.5 \mathrm{~kg} / \mathrm{cm}^{2}$ to a hydrated CG sample equilibrated at $84-\% \mathrm{RH}$. The measuring temperature was changed from -140 to $-23^{\circ} \mathrm{C}$. The sign of electric current is negative at $-140^{\circ} \mathrm{C}$, but becomes positive at $-23^{\circ} \mathrm{C}$. In the temperature range from -73 to $-38^{\circ} \mathrm{C}$, the sign of the current changes from negative to positive, and the positive current shows a maximum.

\section{DISCUSSION}

It is known that elasticity, ${ }^{11-13}$ dielectricity ${ }^{14,15}$ and piezoelectricity ${ }^{9}$ of collagen are influenced by hydration. Baer, et al., ${ }^{12}$ observed three elastic relaxations at $-63,-13$, and $43^{\circ} \mathrm{C}$ at about $1 \mathrm{~Hz}$ for collagen with $35-\mathrm{wt} \%$ moisture content, and those at $-130,-90$, and $-10^{\circ} \mathrm{C}$ for collagen with $10-w t \%$ moisture content. Stefanou, et al., ${ }^{12}$ reported elastic relaxations at -80 and $-20^{\circ} \mathrm{C}$ at $1 \mathrm{~Hz}$ for collagen with $10-\mathrm{wt} \%$ moisture content. Chang, et al., ${ }^{15}$ found dielectric relaxations at $-80,10$, and $88^{\circ} \mathrm{C}$ at $100 \mathrm{~Hz}$ for collagen with $12-w t \%$ moisture content. It appears that the origins of these relaxations have not been well understood. In a previous paper ${ }^{9}$ we proposed tentative interpretations for the piezoelectric relaxations in collagen shown in Figure 1, where maxima of $e^{\prime \prime}$ were seen at $-100,-50$, and $-30^{\circ} \mathrm{C}$. The relaxation around $-100^{\circ} \mathrm{C}$ is associated with the local mode relaxation of main chains of collagen, that around $-50^{\circ} \mathrm{C}$ with the side chain relaxation, and that around $-30^{\circ} \mathrm{C}$ with the increase in ionic conductivity.

The piezoelectricity of bone is believed to be brought on by the piezoelectricity of the crystalline micelle of collagen molecules. ${ }^{16-18}$ The maxima of $e^{\prime \prime}$ around -120 and $-60^{\circ} \mathrm{C}$ for $\mathrm{FB}$ shown in Figure 1 may be associated with the local mode relaxation and the side chain relaxation of collagen respectively. It is noted that $d^{\prime}$ and $e^{\prime}$ for FB and CG reverse their signs at about $-30^{\circ} \mathrm{C}$.

The piezoelectric effect of CG obviously originates in collagen, since the amorphous gelatin layer does not show a piezoelectric effect. The sign of $d^{\prime}$ and $e^{\prime}$ for collagen does not change, but that for CG does change at about $-30^{\circ} \mathrm{C}$. As shown in Figure 4, when a capacitor is connected to the CG film, the temperature of the sign reversal of $d^{\prime}$ shifts to a lower temperature. The effect of the connection of the capacitor is similar to that caused by decreasing the electric capacitance of the gelatin layer. This suggests that the reversal of sign of $d^{\prime}$ and $e^{\prime}$ observed for hydrated CG is caused by the inhomogeneous structure of the three layer system. It can also be supposed that the nonhomogeneous structure of bone consisting of thin spiral layers of collagen fibrils and hydroxyapatite, may cause the inversion of the sign of $d^{\prime}$ and $e^{\prime}$.

As seen in Figures 2, 3, and 5, the variation with temperature of $d, e$, and $\varepsilon$ markedly depends upon the level of hydration. The reversal of sign of $d^{\prime}$ and $e^{\prime}$ is observed for highly hydrated samples (No. 4 and 5), but not for less hydrated samples. At a high temperature range, $\varepsilon^{\prime}$ and $\varepsilon^{\prime \prime}$ for hydrated samples show very large values.

Uemura $^{19}$ proposed that when the influence of ionic impurities is predominant, $\varepsilon^{\prime}$ and $\varepsilon^{\prime \prime}$ are proportional to $f^{-3 / 2}$ and $f^{-1}$ respectively, where $f$ is a measuring frequency. The above relations hold for master curves of $\varepsilon^{\prime}$ and $\varepsilon^{\prime \prime}$ for $C G$ in Figure 7. It is also seen that the temperature range where $d^{\prime}$ and $e^{\prime}$ decrease corresponds to the 


\section{H. UEDA and E. FUKADA}

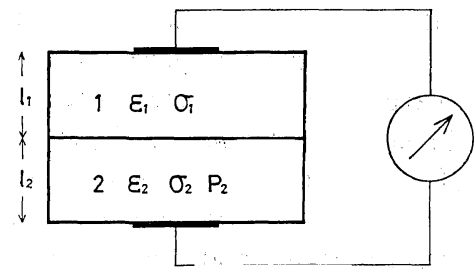

Figure 9. A schematic structure of a combined film of collagen and gelatin.

temperature range where $\varepsilon^{\prime}$ and $\varepsilon^{\prime \prime}$ sharply increase. These observations suggest that ionic conduction is responsible for the decrease and subsequent sign reversal of $d^{\prime}$ and $e^{\prime}$ with increasing temperature observed for hydrated CG.

Figure 9 shows schematically the combined film of gelatin and collagen where layer 1 is the nonpiezoelectric gelatin layer and layer 2 is the piezoelectric collagen layer. In actual samples, gelatin layers are joined to both faces of the collagen layer, but for the sake of simplicity only one gelatin layer is represented in Figure 9. The gelatin layer is joined in series with the collagen layer with respect to the direction of the electric polarization or field. Denoting the thickness of gelatin and collagen layers by $l_{1}$ and $l_{2}$, the dielectric constant of gelatin and collagen by $\varepsilon_{1}$ and $\varepsilon_{2}$, the electric displacement in each layer by $D_{1}$ and $D_{2}$, the electric field in each layer by $E_{1}$ and $E_{2}$, and the piezoelectric polarization of the collagen layer by $P_{2}$, the following relations hold:

$$
\begin{aligned}
& D_{1}=\varepsilon_{1} E_{1} \\
& D_{2}=\varepsilon_{2} E_{2}+4 \pi P_{2}
\end{aligned}
$$

The electric voltage between two electrodes on the surfaces of the samples is $V=l_{1} E_{1}+l_{2} E_{2}$. In our experiments, the piezoelectric polarization is detected under the condition of $V=0$, thus we put

$$
l_{1} E_{1}+l_{2} E_{2}=0
$$

If we assume that there are no true charges in either layer, then

$$
D_{1}=D_{2}=4 \pi q
$$

where $q$ is the surface charge density of the electrodes and equals the polarization, $P$, observed for the sample. From eq $1-3$, the polarization $P$ is given as

$$
P=\frac{l_{2} \varepsilon_{1}}{l_{2} \varepsilon_{1}+l_{1} \varepsilon_{2}} \cdot P_{2}
$$

We denote the piezoelectric stress constant of the collagen layer by $e_{2}$, the strain of the collagen and of the sample by $S_{2}$ and $S$, respectively. Since the layers are combined in series as in Figure 9 and the sample is strained transversely, $S$ is equal to $S_{2}$. Then, using eq 4 the piezoelectric stress constant $e$ of the sample is expressed as,

$$
e=\frac{P}{S}=\frac{P}{P_{2}} \frac{P_{2}}{S_{2}} \frac{S_{2}}{S}=\left(\frac{\alpha \varepsilon_{1}}{\alpha \varepsilon_{1}+\varepsilon_{2}}\right) e_{2}
$$

where $\alpha=l_{2} / l_{1}$ and $e_{2}=P_{2} / S_{2}$.

The piezoelectric strain constant $e$ is related to the piezoelectric stress constant $d$ as follows:

$$
d=e / C
$$

where $C$ is the elastic constant of the sample. After a simple calculation, the $d$ constant obtained is

$$
d=\frac{\alpha \varepsilon_{1}}{\alpha \varepsilon_{1}+\varepsilon_{2}} \frac{1+\alpha}{C_{1}+\alpha C_{2}} e_{2}
$$

where $C_{1}$ and $C_{2}$ are the elastic constants of gelatin and collagen respectively.

It has been shown that the time-temperature equivalence law holds for the piezoelectric and dielectric constants. Increasing temperature corresponds to extending the measuring time. For instance, if the $e$ constant increases with temperature, it also increases with the measuring time. In other words, in a.c. experiments, the phase of polarization lags behind that of applied strain. In such a case, we say that the phase $e$ is lagging and $e^{\prime \prime}$ is positive if the notation $e=e^{\prime}-i e^{\prime \prime}$ is adopted. ${ }^{20}$ If the $e$ constant decreases with temperature, it should decrease with time. In a.c. experiments, the phase of polarization leads that of the applied strain. In such a case the phase of $e$ is leading and $e^{\prime \prime}$ is negative.

According to our observations described above, the reversal of sign of the piezoelectric constant appears to depend upon the conductivity as well as the inhomogeneity of the sample. Usually, the dielectric constant is a function of measuring temperature and angular frequency $\omega$ and is given as a complex quantity. In the present case, the imaginary components of the dielectric constants 
are mainly given by conductivities $\sigma_{1}$ and $\sigma_{2}$, and so we may put $\varepsilon_{1}=\varepsilon_{1}{ }^{\prime}-i\left(4 \pi \sigma_{1} / \omega\right)$ and $\varepsilon_{2}=\varepsilon_{2}{ }^{\prime}-$ $i\left(4 \pi \sigma_{2} / \omega\right)$. Then eq 5 is transformed into

$$
e=\frac{\alpha \varepsilon_{1}{ }^{\prime}}{\alpha \varepsilon_{1}{ }^{\prime}+\varepsilon_{2}{ }^{\prime}}\left(1+\frac{k}{1+i \omega \tau}\right) e_{2}
$$

where

$$
k=\frac{\sigma_{1} \varepsilon_{2}^{\prime}-\sigma_{2} \varepsilon_{1}^{\prime}}{\varepsilon_{1}^{\prime}\left(\alpha \sigma_{1}+\sigma_{2}\right)}=\frac{4 \pi \sigma_{1} \sigma_{2}}{\varepsilon_{1}^{\prime}\left(\alpha \sigma_{1}+\sigma_{2}\right)}\left(\tau_{2}-\tau_{1}\right)
$$

and

$$
\tau=\frac{\alpha \varepsilon_{1}{ }^{\prime}+\varepsilon_{2}{ }^{\prime}}{4 \pi\left(\alpha \sigma_{1}+\sigma_{2}\right)}
$$

where $\tau_{1}=\varepsilon_{1}^{\prime} / 4 \pi \sigma_{1}$ is the dielectric relaxation time for layer 1 , and $\tau_{2}=\varepsilon_{2}{ }^{\prime} / 4 \pi \sigma_{2}$ is the dielectric relaxation time for layer 2, and $\tau$ is the MaxwellWagner relaxation time for the two layers.

Observation of the temperature dependence of $\varepsilon_{1}$ (gelatin) and $\varepsilon_{2}$ (collagen) for samples equilibrated at $84-\% \mathrm{RH}$ shows that $\varepsilon_{1}{ }^{\prime} / \sigma_{1}$ is about one order larger than $\varepsilon_{2}^{\prime} / \sigma_{2}$ below about $-10^{\circ} \mathrm{C}$. Thus we can put $\tau_{1}>\tau_{2}$ and $k<0$ in eq 9 . Equation 8 then shows that $e$ is an increasing function of $\omega$, or is relaxational against measuring time when $e_{2}$ is unchanged with time. From the time-temperature equivalence principle it can be also said that $e$ decreases with increasing temperature when $e_{2}$ is unchanged. Thus the phase $e$ leads that of $e_{2}$.

Figure 1 shows for hydrated collagen (C), now phase 2 , that $e_{2}{ }^{\prime}$ decreases with increasing temperature and $e_{2}{ }^{\prime \prime}$ shows a negative maximum around $-30^{\circ} \mathrm{C}$. In other words, the phase of $e_{2}$ is leading, that is, the polarization $P_{2}$ in the collagen layer is leading in phase with respect to the applied strain $S$. According to the discussion about eq 8, the phase of $e$ is leading with respect to $e_{2}$, that is the observed polarization $P$ is leading in phase with respect to the polarization $P_{2}$ in the collagen layer. As a result of this duplicated effect, the phase of $P$ may lead that of $S$ by more than 90 degrees, which results in the sign inversion of $e^{\prime}$ observed by the a.c. measurement.

The sign reversal of $d^{\prime}$ shown in Figure 2 is also explained in a similar way using eq 7 .

A d.c. bias field $(1.5 \mathrm{kV} / \mathrm{cm})$ was applied to a hydrated CG sample (93-\% RH) at $-2^{\circ} \mathrm{C}$ for $10 \mathrm{~s}$ and the sample was cooled to $-150^{\circ} \mathrm{C}$, then the field was removed and the variation of $d$ constant with increasing temperature was measured. Results are shown in Figure 10. The number indicates

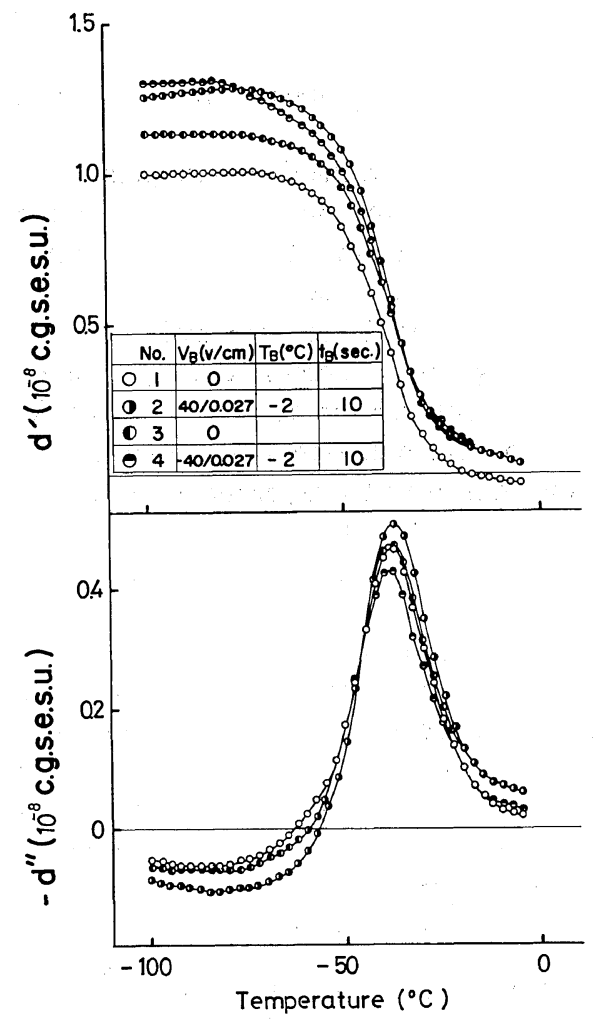

Figure 10. The influence of a d.c. bias field on the temperature dependence of the piezoelectric strain constant $d=d^{\prime}-i d^{\prime \prime}$, for a combined film of collagen and gelatin at $93-\%$ RH.

the sequence of experiments. The direction of the applied d.c. field for No. 2 is opposite to that for No. 4. Curves No. 1 and No. 3 in Figure 10 show the variation of $d$ constant with temperature without the d.c. field.

It is noticed that $d^{\prime}$ for No. 1 reverses sign at about $-20^{\circ} \mathrm{C}$, though $d^{\prime}$ for No. 2-4 involves no sign reverse. The increase of $d^{\prime}$ by applying the d.c. field is not caused by the apparent piezoelectric effect due to the nonhomogeneous distribution of ions, because the data for No. 2 and No. 4 are independent of the direction of applied field. Once a d.c. bias field has been applied, the sign reversal of $d^{\prime}$ is no longer observed. This may be caused by the decrease of the ionic conductivity, $\sigma_{2}$, in the collagen layer, because substantial numbers of ions are swept near the electrodes by the applied field and remain in the gelatin layers. The number of ions swept near the electrodes 


\section{H. UEDA and E. FuKadA}

should depend upon the strength of the applied field, and the temperature at which the field is applied. These effects have been also examined experimentally. ${ }^{21}$

As described above, the time-temperature equivalence holds for the piezoelectric relaxation in polymers, similar to the dielectric and elastic relaxations. ${ }^{10}$ Since the piezoelectric constant measured dynamically for the hydrated CG reverses its sign with increasing temperature, the piezoelectric polarization caused by a static stress should also change its sign with the lapse of time. This was confirmed by experimental results illustrated in Figure 8. The sign of the piezoelectric current at $-140^{\circ} \mathrm{C}$ is opposite to that at $-23^{\circ} \mathrm{C}$. The negative sign of the current implies that the piezoelectric polarization is neutralized by the current inside the piezoelectric collagen phase. The electric current at temperatures from -73 to $-38^{\circ} \mathrm{C}$ changed from negative to positive with time. This indicates that the piezoelectric polarization under a static stress also changes its sign with increasing temperature.

The total polarization reverses sign at the time $t$ at which the relation $\int_{0}^{t_{0}} I \mathrm{~d} t=-\int_{t_{0}}^{t} I \mathrm{~d} t \quad$ is satisfied, where $t_{0}$ is the time of the sign reversal of the piezoelectric current $I$. Figure 11 shows

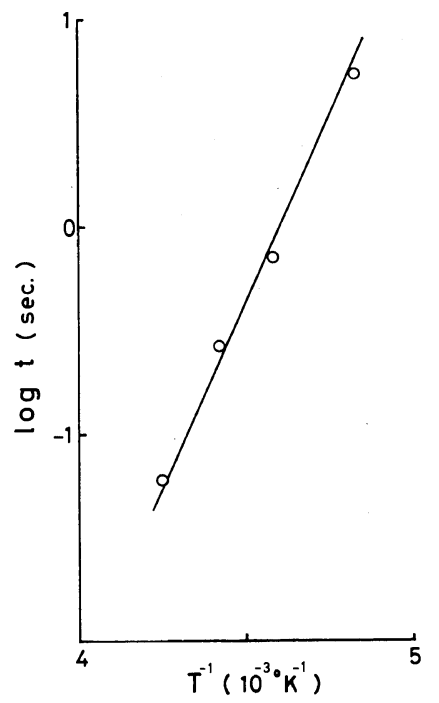

Figure 11. Relation between the reciprocal of the absolute temperature and the time of sign inversion of piezoelectric polarization. the relationship between the reciprocal of the absolute temperature and the time of sign inversion, $t$, determined from the results in Figure 8. It is seen that the time for the sign inversion of polarization becomes shorter with increasing temperature. This accords with the fact that in the dynamical measurement, the temperature of the sign inversion of the piezoelectric constant shifts to higher temperature with increasing measuring frequency.

Now, the piezoelectric polarization of the collagen layer $P_{2}$ under a static stress is a relaxing quantity as discussed already. We assume that $P_{2}$ is given by

$$
P_{2}=P_{0} \mathrm{e}^{-t / \tau_{0}}
$$

where $P_{0}$ is the instantaneous polarization and $\tau_{0}$ the relaxation time. Several possible mechanisms may cause the decay of $P_{2}$ : the increase of the ionic conduction and the elastic relaxation in the boundary region of the piezoelectric phase, and the diffusion of counter ions around dipoles inside the piezoelectric phase, acting to neutralize the polarization. The relaxation time $\tau_{0}$ is not determined by macroscopic conductivity, but $\tau$ and $\tau_{1}$ are determined by conductivities $\sigma_{1}$ and $\sigma_{2}$ in gelatin and collagen layers. We suppose that the decay of $P_{2}$ is most probably caused by the migration of ions or water molecules around oriented dipoles, decreasing the polarization.

The electric current I passing through the layers can be given by

$$
I=\sigma_{1} E_{1}+\frac{1}{4 \pi} \frac{\mathrm{d} D_{1}}{\mathrm{~d} t}=\sigma_{2} E_{2}+\frac{1}{4 \pi} \frac{\mathrm{d} D_{2}}{\mathrm{~d} t}
$$

Using eq 1 to 5 and $12, I$ is expressed as

$$
\begin{aligned}
I= & \frac{\alpha \varepsilon_{1}{ }^{\prime}}{\alpha \varepsilon_{1}{ }^{\prime}+\varepsilon_{2}{ }^{\prime}} P_{0}\left\{\left(\frac{1}{\tau_{1}}-\frac{1}{\tau}\right) \frac{1}{\tau} \mathrm{e}^{-t / \tau}\right. \\
& \left.-\left(\frac{1}{\tau_{1}}-\frac{1}{\tau_{0}}\right) \frac{1}{\tau_{0}} \mathrm{e}^{-t / \tau}{ }_{0}\right\} /\left(\frac{1}{\tau}-\frac{1}{\tau_{0}}\right)
\end{aligned}
$$

Now let us consider a simple case when $\tau_{0}=\infty$ or in other words, the introduction of a fixed amount of polarization $P_{0}$ into the collagen layer (phase 2) due to its piezoelectric effect. Then using eq 10 , eq 13 is modified to

$$
\begin{aligned}
I= & \frac{\alpha \varepsilon_{1}{ }^{\prime}}{\alpha \varepsilon_{1}{ }^{\prime}+\varepsilon_{2}{ }^{\prime}} P_{0}\left(\frac{1}{\tau_{1}}-\frac{1}{\tau}\right) \mathrm{e}^{-t / \tau} \\
& =\frac{\alpha \varepsilon_{1}{ }^{\prime} \varepsilon_{2}{ }^{\prime}}{\left(\alpha \varepsilon_{1}{ }^{\prime}+\varepsilon_{2}{ }^{\prime}\right)^{2}}\left(\frac{1}{\tau_{1}}-\frac{1}{\tau_{2}}\right) P_{0} \mathrm{e}^{-t / \tau}
\end{aligned}
$$


where, as described above, $\tau_{1}$ and $\tau_{2}$ show the dielectric relaxation times of layer 1 and 2 , respectively. If $\tau_{1}<\tau_{2}$, then $I>0$ and if $\tau_{1}>\tau_{2}$, then $I<0$. According to the experimental results it is known that $\tau_{1}>\tau_{2}$ and thus $\tau_{1}>\tau$. Therefore, the negative sign of the current $I$ at very low temperatures accords with this formula.

In an actual case, $\tau_{0}$ is finite and the polarization $P_{2}$ itself will decay with time, as in eq 11 . Thus, we must consider eq 13 .

It is supposed that $\tau_{0}$ is large at low temperatures and decreases with an increase in temperature. If $\tau_{0}$ has a very large value, eq 13 approaches eq 14 . Under the condition of $\tau_{0}>\tau_{1}>\tau$, both the first and the second terms in eq 13 are negative and give a decay curve of negative current, which is similar to the piezoelectric current observed at $-140^{\circ} \mathrm{C}$ in Figure 8. If $\tau_{0}$ has a moderate value and the condition $\tau_{1}>\tau_{0}>\tau$ holds, then the first term in eq 13 is negative and the second term is positive. Then eq 13 gives a curve with a sign reversal, which is similar to the piezoelectric currents observed at intermediate temperatures in Figure 8. If $\tau_{0}$ is very small such as $\tau_{1}>\tau>\tau_{0}$ the first term in eq 13 is positive and the second term is negative but very small. Equation 13 gives a decay curve of positive current, which is similar to the curve observed at $-23^{\circ} \mathrm{C}$ in Figure 8. At intermediate temperatures from -73 to $-38^{\circ} \mathrm{C}$ the current to neutralize the piezoelectric polarization $P_{2}$ and the current due to the decay of $\boldsymbol{P}_{2}$ flow in opposite directions. Since the magnitude of their relaxation times $\tau$ and $\tau_{0}$ are different, the superposition of the two decay curves yields a reversal in sign with time as shown in Figure 8.

To summarize, the sign of the piezoelectric constant is determined by the direction of current in the sample. The sign inversion of the piezoelectric constant with increasing temperature can be explained by considering two factors. One is the flow of current to neutralize the piezoelectric polarization in the inhomogeneous structure with the relaxation time $\tau$ and the other is the decay of piezoelectric polarization in the collagen layer with the relaxation time $\tau_{0}$.

Bone consists of oriented collagen fibers and hydroxyapatite crystallites, which can be approximated as a two phase composite system. Since collagen absorbs more moisture and shows a higher conductivity compared to hydroxyapatite, the ex- planations used for the combined system of collagen and gelatin can be similarly applied to the sign inversion of the piezoelectric constants of bone.

Acknowledgement. The authors wish to thank Dr. M. Date for his useful comments. This work was supported by a research grant for the Studies of Life Science at the Institute of Physical and Chemical Research.

\section{REFERENCES}

1. H. Fröhlich and R. Sack, Proc. Roy. Soc., A185, 415 (1946).

2. K. W. Wagner, Arch. Elektrotech., 2, 371, 374, 383 (1914).

3. R. Hayakawa and Y. Wada, Rep. Prog. Polym. Phys. Jpn., 15, 373 (1972)

4. M. Date, Polym. J., 8, 60 (1975).

5. H. Ueda and E. Fukada, Rep. Prog. Polym. Phys. Jpn., 18, 367 (1975).

6. H. Lenormant, A. Baudras and E. R. Blout, $J$. Am. Chem. Soc., 80, 6191 (1958).

7. M. H. F. Wilkins, Science, 140, 941 (1963).

8. Y. Ando and E. Fukada, J. Polym. Sci., 14, 63 (1976).

9. E. Fukada, H. Ueda, and R. Rinaldi, Biophys. J., 16, 911 (1976).

10. R. Furukawa and E. Fukada, Rep. Prog. Polym. Phys. Jpn., 16, 457 (1973); J. Polym. Sci. Phys., 14, 1979 (1976).

1. A. Tanioka, E. Tojima, K. Miyasaka and K. Ishikawa, J. Polym. Sci., 11, 1489 (1973).

12. H. Stefanou, A. E. Woodward, and D. Marrow, Biophys. J., 13, 772 (1973).

13. E. Baer and R. Kohn, J. Macromol. Sci. Phys., B6(4), 761 (1972).

14. V. P. Tomaselli and M. H. Shamos, Biopolymers, 12, 353 (1973).

15. E. P. Chang and J. C. W. Chien, J. Polym. Sci., 11, 737 (1973).

16. E. Fukada and I. Yasuda, J. Phys. Soc. Jpn., 12, 1158 (1957).

17. C.A. Bassett and R.O. Becker, Science, 137, 1063 (1962)

18. J. C. Anderson, C. Eriksson, Nature, 227， 491 (1970).

19. S. Uemura, J. Polym. Sci., 10, 2155 (1972).

20. E. Fukada and M. Date, J. Macromol. Sci., B8, 463 (1973).

21. H. Ueda and E. Fukada, Rep. Prog. Polym. Phys. Jpn., 18, 605 (1975). 